\title{
Accessibility measures for robustness of the transport system
}

\author{
Feixiong Liao' ${ }^{1}$ Bert van Wee $^{2}$
}

Published online: 17 May 2016

(C) The Author(s) 2016. This article is published with open access at Springerlink.com

\begin{abstract}
Accessibility is a key concept in transport and land use policies, and infrastructure-based measures are one important category of accessibility measures. Recently, there has been a significant increase in the attention paid in both academic literature and policy documents to the robustness of the transport system. However, there is not a mature body of literature on the infrastructure-based accessibility measures expressing this concept. This paper proposes a family of accessibility measures to express the robustness of the transport system. These have in common that they express the number of travel options between a given origin and destination, or to conduct activities. The family of measures is conceptualized by using the multi-state supernetwork approach. With respect to the travel options, we discuss (1) the overlap of travel routes, and (2) the choices of travel modes (e.g. car versus train; multimodal). With respect to the activities, we discuss (1) the choice of OD pairs as opposed to activity-travel patterns, and (2) fixed versus flexible activity locations and fixed versus flexible order of activities. We illustrate the travel options and robustness in a multi-modal transport system between the city centers of the HagueRotterdam-Dordrecht corridor (The Netherlands).
\end{abstract}

Keywords Accessibility · Travel options · Route overlap · Multi-state supernetwork

Bert van Wee

g.p.vanwee@tudelft.nl

1 Department of the Built Environment, Eindhoven University of Technology, PO Box 513, 5600 MB Eindhoven, The Netherlands

2 Transport and Logistics Group, Delft University of Technology, PO Box 5015, 2600 GA Delft, The Netherlands 


\section{Introduction}

Accessibility is a key concept for evaluating the performance of the transport system, and consequently a major concept for transport policies. Following Geurs and Van Wee (2004) and limiting ourselves to passenger transport, we define accessibility as 'the extent to which land-use and transport systems enable individuals to reach activities or destinations by a (combination of) transport mode(s)'. We use the term 'measures' for the general expression of accessibility, and the term 'indicator' for a specific operationalization of a measure. Geurs and van Wee (2004) gave an overview of families of measures, one category being infrastructure-based measures described as 'analyzing the (observed or simulated) performance or service level of transport infrastructure, such as level of congestion and average travel speed on the road network. This measure type is typically used in transport planning'.

Recently, there has been rapidly growing awareness that the generalized travel costs (GTC) should not only include travel time, monetary costs, and trouble/comfort, but also the predictability/variability of travel times. Related terms include robustness, reliability, vulnerability, flexibility, and resilience, etc. We firstly provide a few definitions. Reggiani et al. (2015) stated that 'resilience analysis refers to the speed at which-and the pathway along which-a network returns to its equilibrium after a shock, as well as to the perturbations/shocks that can be absorbed', and that 'vulnerability analysis refers to the susceptibility to extreme strains on a dynamic system'. Cats and Jenelius (2015) provided a definition of the robustness of a public transport system which can easily be generalized to the transport system: it is 'the ability to withstand or quickly recover from disturbances such as infrastructural and vehicular malfunctions and planned maintenance closures without significant reduction in the performance of the system (in terms of travel times etc.)'.

We next give a brief overview of the literature in this area. Bates et al. (2001) discussed the theoretical and empirical literature of the valuation of travel reliability. Lam and Small (2001) developed models based on empirical results concluding that the value of reliability is $\$ 15.12$ per hour for men and $\$ 31.91$ for women. Rietveld et al. (2001) concluded that users of public transport (PT) have a strong attitude of risk aversion towards travel time. Chen et al. (2002) proposed a method to assess the capacity reliability of road networks. Mazloumi et al. (2010) estimated day-to-day variability in bus travel times using GPS data. Börjesson and Elisasson (2011) studied how long-distance train passengers value unexpected delays, and concluded that the disutility increases more slowly than linearly in the delay risk. Koopmans et al. (2013) proposed an indicator that also includes the reliability of travel times. In the editorial of a special issue in Transportation Research part A on "resilience of networks", Caschili et al. (2015) provided definitions and explain how some of these concepts are related. These concepts have also been included in some policy documents. For example, the Dutch government aims to increase the reliability of travel times on roads, waterways, and railways (Ministerie van Infrastructuur en Milieu 2012).

The variability of travel times is linked to the more general concept of the robustness of the transport system, which is the opposite to the vulnerability of it. This study proposes an indicator of the robustness of the transport system associated with the number of options that a traveler has to travel either between given origins and destinations, or to carry out given activities. The motivation for this proposal is that the more options travelers have, the less vulnerable they are to disturbances in the transport system, such as delays due to road accidents, or substantial train delays. We find that this is not the only possible 
indicator for robustness, just as that any accessibility indicator proposed in the literature is not the only one in the same family of accessibility indicators. And we realize others might prefer another term than 'robustness' - the debate on terminology is not closed yet. Note that travel options between activity locations so that people can fulfill activity programs are the core of time geography measures (e.g. Neutens et al. 2007), and that the identification of alternative routes is common in route choice and path choice modeling (e.g., Bekhor et al. 2006; Bovy and Fiorenzo-Catalano 2007) — these elements in themselves are thus not new. What is new in our proposal is to present travel options as a robustness indicator.

Conceptually, the availability of more travel options matters for two major reasons. Firstly, individuals value the reduced travel time variability that results from having more options. If, for example, there is a road block somewhere in the road network and an alternative route is available with hardly a detour nor capacity limitations, the road block will not affect the travelers. The availability of multiple options and the impact of the road block on travel times/GTC might occur both preceding the trip (e.g. being aware of problems in part of the rail network whereas alternatives are available) and during the trip (e.g. dynamic satellite navigation can suggest an alternative route because of unexpected congestion on the standard route). Secondly, individuals in general value having multiple travel options, regardless of their actual use - they may value the potential of having these options, as recognized in the concept of the option value (Geurs et al. 2006; Laird et al. 2009).

However, despite the increasing attention paid to topics like robustness, vulnerability, and travel time variability in the academic literature, to the best of our knowledge no dedicated family of accessibility measures for the robustness of the transport system exists, although there are some examples of indicators that include reliability (e.g., Koopmans et al. 2013). Koopmans' indicator is a GTC indicator including reliability to some extent, but it is not an indicator explicitly addressing the concept of the availability of multiple travel options. The popular contour measure (number of destinations accessible within a threshold value, generally expressed in distance, time or GTC) or potential accessibility measures (number of destinations accessible, whereby the relevance decreases with the increase of distance, costs, or GTC) can be seen as measures for the provision of alternative destinations. One could argue that a higher level of the potential accessibility indicator expresses more options to travel for specific trips. This holds for trips that in a short term (e.g. daily or hourly basis) have flexibility regarding destination choice, such as daily shopping, but not for trips with fixed locations, such as most work-related trips. It might seem that the logsum accessibility measures (e.g. De Jong et al. 2007) value travel options, but it is important to realize that the inclusion of multiple options only results from uncertainty on the side of the modelers (Chorus and De Jong 2011), and is not meant to measure the options offered by the transport system. It is also important to realize that the logsum measures only express the value of the options and is therefore not necessarily an indicator which directly expresses accessibility. Similarly, D'Lima and Medda (2015: 35) proposed a new measure for resilience, following Pimm (1991) defined as "how fast a variable that has been displaced from equilibrium returns to it". Janic (2015) proposes a methodology for the "ability of the network to neutralize the impacts of disruptive event(s)". Although related to our work, their measures/approaches do not focus on the number of opportunities available for travelers.

Therefore, the aim of this paper is to propose a family of measures for the robustness of the transport system. More specifically, the measures express the number of travel opportunities (either weighted by a cost function or not) available for the travelers. We mainly use examples in the area of passenger transport, but the measures could be used in a 
comparable way for freight transport. We limit ourselves to physical accessibility, largely ignoring Information and Communication Technologies (ICT)-based accessibility (Van Wee et al. 2013). The proposed accessibility measures not only fill a gap in the literature, but are also relevant for policy-making. Policymakers might be interested in the number of travel options people and companies have, because it tells them how vulnerable the transport system is for disruptions. In many countries, large disruptions receive a lot of negative media attention, which is an indication for the societal relevance of the topic. By making the transport system more robust (via increasing the number of travel options) policy makers can increase welfare because travel time losses due to disruptions are valued negatively (see above), even more than expected travel time losses due to recurrent congestion. Including the robustness in ex ante evaluations of infrastructure options and next decision-making could, for example, lead to deciding to prioritize parallel road infrastructure options over improving the capacity of one single motorway.

So we argue policy makers can benefit from our indicators and the applications are manifold, including:

- Comparisons between geographical areas of the robustness of the transport system. Such comparisons can reveal in which regions the transport system is relatively vulnerable.

- Comparisons of the robustness of an area over time. Such comparisons can reveal trends, e.g. due to changes in congestion levels or land-use patterns.

- Impact of policy measures on robustness. Of particular interest are infrastructure policies, non-infrastructure related transport policies (e.g. related to time tables of PT, road pricing), effects of changing opening hours of location services, and synchronization policies, etc. Synchronization increasingly receives attentions in the academic literature. The general idea is that synchronization between land use and the transport system results in benefits in several areas, such as increased travel comfort, reduced travel times, increased accessibility, and maybe also increased travel options. Van Wee et al. (2014) refer to examples of synchronization in the areas of PT networks (Guihaire and Hao 2008; Guo and Wilson 2011), land use and transport planning (Levine 2005; Bertolini et al. 2012), teleworking and travel (Mokhtarian 2002) and multitasking while travelling (Lyons and Urry 2005).

The remainder of this paper is organized as follows. "The proposed family of measures" section proposes the family of measures and discusses important features of the measures based on the conceptualizations needed to operationalize the measures. "Operationalization of the measures" section proposes a methodology to operationalize the measures. "Illustration: a case study" section illustrates the operationalization in a case study in a multi-modal transport system. "Conclusions and future work" section finally presents the main conclusions and discusses avenues for further development of the measures, valuation of outcomes, and their use for policy.

\section{The proposed family of measures}

Our measures for the robustness of the transport system are based on the potential accessibility measures, but we reverse the land use component and the transport system component. Robustness is equal to the potential number of travel options available for (1) either given origins or destinations, or (2) given activity programs and including flexibility 
with respect to the choice of the destination and even the origin. These travel options can be calculated using simple cut-off values, e.g., only options with travel times less than $130 \%$ of the fastest option, comparable to contour measures, or travel options can be weighted using travel time or GTC, comparable to potential accessibility measures. Below we further discuss the conceptualizations of the measures. We first explain alternatives for travel options only ("Travel options" section), followed by a discussion on multiple origins and destinations ("Origins, destinations, activities" section).

\section{Travel options}

\section{(1) What is an alternative travel option?}

An important question is: what is an alternative travel option? Or in other words, which options should be counted? Ideally, travel options should be uncorrelated. However, options often have a degree of overlap. For example, multiple routes available for a car trip, a PT trip, or a multimodal trip generally have a degree of overlap. This problem was discussed in the context of route choice (Prato 2009), but needs to be further developed for the proposed family of measures. A simple method could be that the overlap in travel options is corrected by adding a factor expressing the extent to which options are unique. The point of departure is the 'best' travel option, which is fully taken into account using the value of 1.0. Sequentially, if an alternative travel option have no overlap with the identified options, the factor is 1.0 and the alternative counts as a full alternative; in case of some overlap, the factor decreases. Then, an important choice to be made is: how can the overlap best be expressed? Overlap relates to the segments of the routes which are identical. The overlap could be expressed in terms of any cost function, but travel time or GTC are the most relevant. In case a cut-off value is included, as mentioned above, both the value and the variable needs to be chosen.

\section{(2) Which mode(s) to include?}

Similar to conventional potential accessibility measures, a choice needs to be made with respect to which modes to be included. The choice can be made to focus on one mode only (car, PT, walking, cycling, aircraft, etc.), but multimodal trips should also be considered. If only one vehicle is considered, options may only differ with respect to routes or departure time in the dynamic setting.

\section{(3) Mathematical expression}

By assuming "distance decay", we can specify the indicator using Eq. 1:

$$
\text { Acc_Robustness } s_{s}=\sum_{t} \sum_{k} \text { factor_overlap }_{k} \times f_{k}\left(C_{s t}\right)
$$

where Acc_Robustness $s_{s}$ is the value of the accessibility indicator for robustness for a given zone/origin $s, t$ is one of the destination zones, factor_overlap $k$ is the factor correcting for overlap between a travel option $k$ and all other travel options, and $f_{k}\left(C_{s t}\right)$ is the cost function for travel option $k$ expressing that more remote destinations account less. 


\section{Origins, destinations, activities}

Another important question is: what are 'given' in the measures, origins and destinations (ODs) or activities? For given origins or destinations, the measures have strong similarities with contour and potential accessibility measures. However, the measure in our case expresses the number of travel options for one or multiple ODs, which can be defined:

1. At the level of only one OD pair.

2. Over a number of potential destinations from one given origin.

3. Over a number of origins for a given destination.

4. Over a number of origins and a number of destinations.

Option 1 is probably only relevant for individual choices/evaluations, such as persons who want to know how they can travel to the airport, or a company that wants to know the travel options between different locations (e.g. distribution centers). The same applies to options 2 and 3, but travel options for multiple destinations are included. These options can be relevant, for example, for companies selecting their office location. They may be interested in options to travel to clients, or in options for clients or employees to visit their offices. Option 4 is the most relevant from the policy perspective as policies need to evaluate the travel options over a larger area, including many OD pairs.

For given activity programs, the measures express the number of travel options for carrying out them. A particular activity pattern, for example, could be that a traveler wants to combine work, shopping, and visiting friends in one chain. Some activity locations are fixed, while others are not. For example, most work activities are carried out at fixed locations but supermarkets can be easily substituted (multiple locations). Some activities have time constraints (e.g. business meeting), whereas others are more flexible (e.g. shopping). Some activities need to be carried out in a specific order (e.g. children need to be brought to school before going to work, shopping precedes bringing the goods bought home). In this case, the choice needs to be made whether activity locations are assumed to be fixed or not, and whether the order of activities is fixed or flexible, allowing four options:

1. Fixed locations, fixed order of activities.

2. Fixed locations, flexible order of activities.

3. Flexible locations, fixed order of activities.

4. Flexible locations, flexible order of activities.

These four options do not reflect the whole spectrum of options. Firstly, the level of substitutability of locations varies between activities, and the distinction between fixed and flexible locations is better conceptualized as a continuum rather than a $0-1$ variable. For example, some work needs fixed locations, but not all. Some work activities can be done at multiple locations making use of ICT, whereas others need fixed locations. Secondly, time constraints and the order of activities can vary in terms of flexibility. For example, shopping can be done at any time whilst the shops are open, but if one buys food that needs to be cooled one cannot leave it in the car all day. Picking the children up from kindergartens can generally be done within a certain time interval, allowing for more flexibility. We argue that the behavioral realism of the measures increases if the measures allow for flexibility in activity frequency, locations, durations and orders. On the other hand, this increased behavioral realism comes with important costs, the first one being computational effort, and the second being the ease of communication of results-a trade-off that applies to many accessibility measures (Geurs and Van Wee 2004). 
This paper focuses on proposing measures at a conceptual level, providing specific solutions to be generally applied for the choices is beyond its scope. We nevertheless propose possible solutions in "Operationalization of the measures" and "Illustration: a case study" sections as an illustration. We believe that as constraints related to activity participation become more present, the more these measures can make use of principles developed in time-space geography (Neutens et al. 2007).

\section{Operationalization of the measures}

This section proposes a methodology to operationalize the measures based on network representations. "Travel options for fixed origins and activity locations" section presents the methodology for traveling to a fixed activity location, "Travel options for a flexible activity" section for flexible activity locations, and "Travel options for an activity program" section for activity programs.

\section{Travel options for fixed origins and activity locations}

Below we propose the methodology for travel from a fixed origin (e.g. home) to a fixed activity location (e.g. work), resulting in a fixed O-D pair. We discuss travel by a private vehicle, public transport and as well as multimodal travel.

\section{Private vehicle $(P V)$}

PVs can be car and (e-)bike. Travel options are alternative routes between the O-D pair. We assume that departure or arrival times are fixed. Each alternative has generalized travel costs (GTC), a mode-specific function which includes travel time, travel distance, and monetary cost, etc. GTCs can be calculated in a weighted transport network. Let $s$ and $t$ denote the origin and the destination respectively, $P_{i}$ a path from $s$ to $t$, and $C\left(p_{i}\right), T\left(p_{i}\right)$, $D\left(p_{i}\right), \operatorname{GTC}\left(p_{i}\right)$ the associated cost, time, distance and GTC of $p_{i}$ respectively. In order to qualify travel option candidates, we need to set upper bounds for the maximum GTC or GTC components, and the maximum level of overlap between options.

Upper bound for GTC or GTC components There are numerous routes between two locations, especially at the urban and regional scale. Including all options is computationally burdensome and meaningless. A selection can be based by taking the route with the lowest GTC or GTC components as a point of departure. Let $p^{*}$ denote the best route option. Next, the upper bound needs to be set, expressed as a factor by which $p^{*}$ needs to be multiplied. For example, an individual may not consider a path with costs more than $2 \times C\left(p^{*}\right)$ as an alternative. The upper bound can be set in terms of costs, time, distance, or GTC (Eqs. 2-5).

$$
\begin{gathered}
C\left(p_{i}\right) \leq f_{C} \times C\left(p^{*}\right) \\
T\left(p_{i}\right) \leq f_{T} \times T\left(p^{*}\right) \\
D\left(p_{i}\right) \leq f_{D} \times D\left(p^{*}\right) \\
\operatorname{GTC}\left(p_{i}\right) \leq f_{G} \times \operatorname{GTC}\left(p^{*}\right)
\end{gathered}
$$

where $f_{C}, f_{T}, f_{D}, f_{G}$ are the upper bound factors. 
Upper bounds for overlap in paths The $K$-shortest path algorithm (Yen 1971) is often adopted to find the first $K$ lowest cost paths with the upper bounds specified above. However, it is quite common that there is only a minor difference in routes between the $k$ th and $k+1$-th path, which results in the so-called path overlapping problem. A common way is to exclude paths with only minor differences. Given two paths $p_{i}$ and $p_{j}$ $\left(0<C\left(p_{i}\right) \leq C\left(p_{j}\right)\right)$, let $P O\left(p_{i}, p_{j}\right)$ denote the path segments of $p_{i}$ that belong to $p_{j}$. The path overlapping ratio in terms of cost $R_{C}\left(p_{i}, p_{j}\right)$ between $p_{i}$ and $p_{j}$ can be expressed as:

$$
R_{C}\left(p_{i}, p_{j}\right)=\frac{C\left(P O\left(p_{i}, p_{j}\right)\right)}{C\left(p_{i}\right)}
$$

$R_{C}\left(p_{i}, p_{j}\right)=0$ means no overlap, whereas if $R_{C}\left(p_{i}, p_{j}\right)$ approaches one, there is a very large overlap. As an illustration, Fig. 1 displays a simple transport network, where nodes and curves denote locations and road connections respectively. Suppose $p^{*}=p_{1}=s \rightarrow a \rightarrow b \rightarrow c \rightarrow t, \quad p_{2}=s \rightarrow a \rightarrow c \rightarrow t$ and $p_{3}=s \rightarrow b \rightarrow t$ satisfying $0<C\left(p_{1}\right) \leq C\left(p_{2}\right) \leq C\left(p_{3}\right)$; then, we have $P O\left(p_{1}, p_{2}\right)=s \rightarrow a+c \rightarrow t$ and $R_{C}\left(p_{i}, p_{j}\right)=$ $\frac{C(s \rightarrow a)+C(c \rightarrow t)}{C\left(p_{i}\right)}, P O\left(p_{1}, p_{3}\right)=\emptyset$ and $R_{C}\left(p_{1}, p_{3}\right)=0$.

Let $P^{\mathrm{S}}$ denote the set of identified travel alternatives and $p_{k}^{s}$ the $k$-th element of $P^{\mathrm{S}}$, with $C\left(p_{l}^{s}\right) \leq C\left(p_{k}^{s}\right)$ to $\forall l<k$. Clearly, we have $p_{1}^{s}=p^{*}$. Paths to be included should not overlap more than set in the upper bound ratio:

$$
R_{C}\left(p_{i}, p_{j}\right) \leq r_{C} \text { to } \forall p_{i} \in P^{\mathrm{S}}
$$

where $r_{C}$ is the upper bound ratio for path overlap in terms of cost.

Similarly, the upper bound inequalities in terms of time, distance and GTC are expressed by substituting the subscripts in Eq. 7:

$$
\begin{aligned}
& R_{T}\left(p_{i}, p_{j}\right) \leq r_{T}, \text { to } \forall p_{i} \in P^{\mathrm{S}} \\
& R_{D}\left(p_{i}, p_{j}\right) \leq r_{D}, \text { to } \forall p_{i} \in P^{\mathrm{S}} \\
& R_{G}\left(p_{i}, p_{j}\right) \leq r_{G}, \text { to } \forall p_{i} \in P^{\mathrm{S}}
\end{aligned}
$$

By checking Eqs. 2-5, 7-10 constantly for any newly generated path candidate, the selection from the $K$-shortest paths, which are included for the robustness indicator, can be derived. The $K$-shortest path algorithm traditionally finds the $K$ - shortest paths sequentially. However, they do not necessarily satisfy the inequalities defined above. Thus, every newly generated path after the initial one should be checked for its validity as a travel option. This process continues until no valid travel option can be obtained. Theoretically, finding the $K$ shortest path under constraints (Eqs. 2-5, 7-10) is a NP-hard problem (non-deterministic polynomial-time), which means that an algorithm with polynomial run-time does not exist. In other words, it is computationally burdensome to find all the valid travel options. However, approximate algorithms (Prato 2009) are acceptable to find the near-optimal

Fig. 1 Example of a PV network

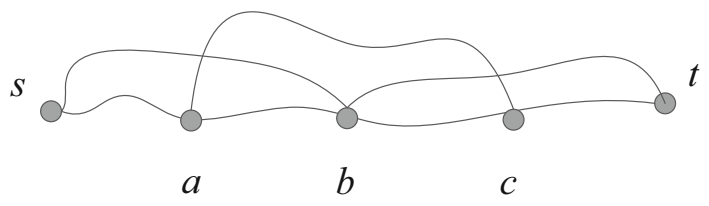


solutions by designing refined link elimination mechanisms. For example, a mechanism for regional travel is to eliminate links at higher levels in a hierarchical road network, where road types are differentiated in terms of speed limit. For regional trips, travelers tend to travel on the roads of hierarchical structure local $\rightarrow$ regional $\rightarrow$ highway $\rightarrow$ regional $\rightarrow$ local. Travelers consider travel options by first substituting the highway, then regional and lastly the local road. Based on the two upper bounds, we can find an approximate set of travel options. In a three-level hierarchical road network with highway and local-road as the highest and lowest level respectively, the pseudo-code of the algorithm can be described as follows:

Step 1: insert the path with the lowest cost in the set of travel options, i.e., $P^{S}=\left\{p^{*}\right\}$, and insert $p^{*}$ in the set of active paths for generating new travel options, $P^{A}=\left\{p^{*}\right\}$.

Step 2: select the first element $p_{1}^{A}$ of $P^{A}$, first eliminate links of the highest level in $p_{1}^{A}$ and find the temporary lowest cost path $p^{* \prime}$. If $p^{* \prime}$ satisfies the upper bounds, insert $p^{* \prime}$ into $P^{\mathrm{S}}$ and at the end of $P^{A}$. Otherwise, if only path overlap upper bound is not satisfied, further eliminate links of the second highest level in $p_{1}^{A}$, find $p^{* \prime}$. If $p^{* \prime}$ satisfies the upper bounds, insert $p^{* \prime}$ into $P^{\mathrm{S}}$ and at the end of $P^{A}$; otherwise, continue to eliminate links of local-road level.

Step 3: remove $p_{1}^{A}$ from $P^{A}$. If $P^{A}$ is empty, the algorithm stops; otherwise, go to step 2.

Step 4: output $P^{\mathrm{S}}$ as the set of travel options.

The travel options are sorted from the smallest to the largest in terms of the main measure of travel options. We can then specify the indicator of travel robustness using Eq. 11:

$$
a c c_{s t}^{r}=\exp \left(-\frac{C\left(p^{*}\right)}{\beta_{B}}\right)+\sum_{k>1} \exp \left(-\frac{C\left(p_{k}\right)}{\beta_{B}}\right) *\left(1-\max \left\{R_{C}\left(p_{l}, p_{k}\right), \forall l<k\right\}\right)
$$

where $a c c_{s t}^{\mathrm{r}}$ denotes the travel robustness for an $s-t$ pair. The term ' $1-\max \left\{R_{C}\left(p_{l}, p_{k}\right), \forall l<k\right\}$ ' corrects for the maximum overlap between any additional path and the already-selected ones. The parameter $\beta_{B}$ rescales the value of the accessibility indicator. $\beta_{B}$ represents the maximum value in terms of the main measure (time, cost, or GTC, etc.) acceptable to individuals to travel between any OD pairs in the study area. If the indicator is used to compare accessibility in one region for the same type of destinations (e.g. access to hospitals in an area), the value can be set more or less arbitrarily because all the alternatives are corrected equally. If a comparison encompasses different areas and people have different attitudes on acceptable travel times or costs, the values may differ between those areas and different groups of people, and these values should preferably be based on empirical research. If travel time is considered the main measure for evaluating travel options, $\beta_{B}$ is set as the maximum shortest travel time per trip in the study area.

The indicator includes the travel options available and corrects for overlap: the more overlap, the less the contribution of an alternative to the value of the robustness indicator. It also includes an exponential form to decrease the additional contribution to the value of the indicator as the costs of available options increase. In such a way, the indicator is linked more to the utility of the robustness indicator than if simply summing the options only corrected for overlap.

So far we have presented the methodology for private vehicles. This methodology can be applied to PT networks, multimodal travel, and activity programs, by conceptualizing PT, multimodal travel and activity programs as generic networks. Therefore, the methodology and formula can be used in the same way as for private vehicles. 


\section{Public transport $(P T)$}

Travel options with PT are less discussed in the literature compared to private vehicle (PV) options. If the PT system is expressed in terms of a network, the calculation of the PT travel options is more or less the same as for PV options. As suggested by Pyrga et al. (2008), timetable-based PT schedules can be represented as time-expanded networks, detailing the full link information of in-vehicle, waiting and transfer between different PT routes. Figure 2 presents an example of an $s-t(\mathrm{O}-\mathrm{D})$ trip via a PT network, including access and egress and PT travel (taken from Liao et al. 2011), in which transfer and waiting occurs at the same PT stops between different PT routes. A PT trip may involve a combination of multiple PT modes, such as bus, train, and tram etc.

Because the PT system is represented in network terms, we can find $K$ travel options with PT. Likewise, the PT network can be constructed in a hierarchical fashion, with PT links downgraded from intercity train, via metro/subway, to local bus. Therefore, the pseudo-code proposed above can also be adapted to find the travel options.

Depending on data availability, the calculation of the number of PT travel options can consider the number of independent tracks (e.g. a slow train network and a high speed network), the number of line routes or the number of connections/services. In case of a breakdown of one network link the number of track options determines the robustness. In case of the breakdown of one single train, the number of alternative services is important.

In this study, the timetable of public transport is considered for finding the travel options, but the frequency of public transport is not incorporated in Eq. 11.

\section{Travel options of multi-modal trip}

We define a multi-modal trip as the usage of a combination of PV plus PT. Such a multimodal trip can be represented in a so-called augmented network or supernetwork (Carlier et al. 2003; Liao et al. 2010, 2016). Let a pentagon denote a PV network (PVN) that can only be accessed by private vehicles, i.e. the bike or car, let a hexagon denote a PT network (PTN) that can be accessed by foot and other PT modes, and let vertices denote locations. Figure 3 illustrates a multi-modal transport network. $P_{1}$ and $P_{2}$ are two potential parking locations. $P_{1}^{\prime}$ and $P_{2}^{\prime}$ are the same locations as $P_{1}$ and $P_{2}$ respectively and denote that the private vehicle is parked. Thus, any path from $s$ to $t$ represents a multi-modal trip chain.

Parking location options can be easily included in the network by adding link costs (GTC) on the parking links, e.g. $P_{1} \rightarrow P_{1}^{\prime}$ and $P_{2} \rightarrow P_{2}^{\prime}$. Thus, the lowest cost path from $s$

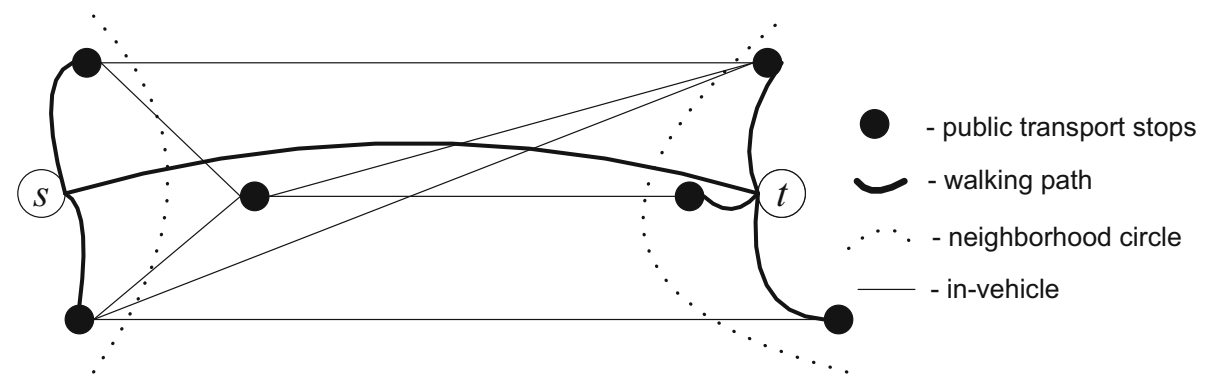

Fig. 2 Example of a PT network 
Fig. 3 Example of multi-modal trips

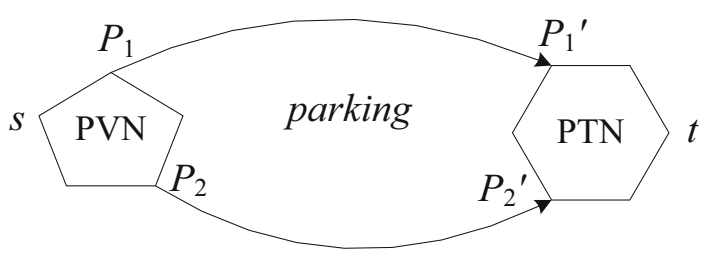

to $t$ is still regarded as the first reference for a travel option. Equations $2-5$ and $7-10$ can then be used to delineate travel options for multi-modal trips. A parking location, other than the optimal parking location (that causes the least cost), may be included as part of a travel option, which potentially cause less overlap if those two parking locations are far away. Thus, the links representing parking location choice (Fig. 3) can be viewed as links with the highest level in the hierarchical network.

\section{Travel options for a flexible activity}

Likewise, we can represent the travel options for an activity that can be conducted at one of multiple locations, such as one of the supermarkets for shopping. Figure 4 illustrates a flexible activity with two alternative locations, $A_{1}$ and $A_{2}$. Although travel options now also involve (activity and parking) location choice, they can still be represented by defining the links cost (GTC) on activity links, i.e. $A_{1} \rightarrow A_{1}{ }^{\prime}$ and $A_{2} \rightarrow A_{2}{ }^{\prime}$ (Liao et al. 2010). The lowest cost path from $s$ to $A_{1}{ }^{\prime}$ or $A_{2}{ }^{\prime}$ stands for the most attractive travel option. Links representing conducting activity can also be viewed as links with the highest level in the hierarchical network. Equations $2-5$ and $7-10$ can be adjusted to identify travel options and derive the robustness for conducting the activity using Eq. 11.

\section{Travel options for an activity program}

With the concept of a multi-state supernetwork (Arentze and Timmermans 2004), we can define the travel options for conducting an activity program that potentially involves multimodal and multi-activity trip chaining. The implementation of a full activity program is represented as a path through a constructed supernetwork, and travel options can then be calculated in the supernetwork. Figure 5 shows the network representation for conducting an activity program for a fixed sequence of two fixed activities using PT, first $A_{1}$ and then

Fig. 4 Example of multi-modal trip for flexible activity

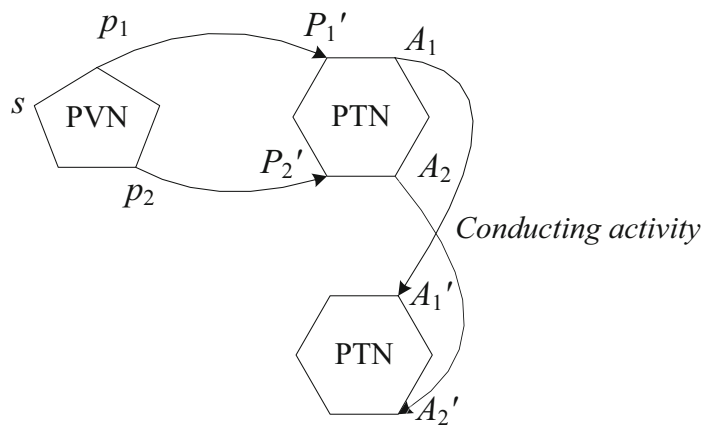


Fig. 5 Example of an activity program with a fixed sequence (taking PT only)
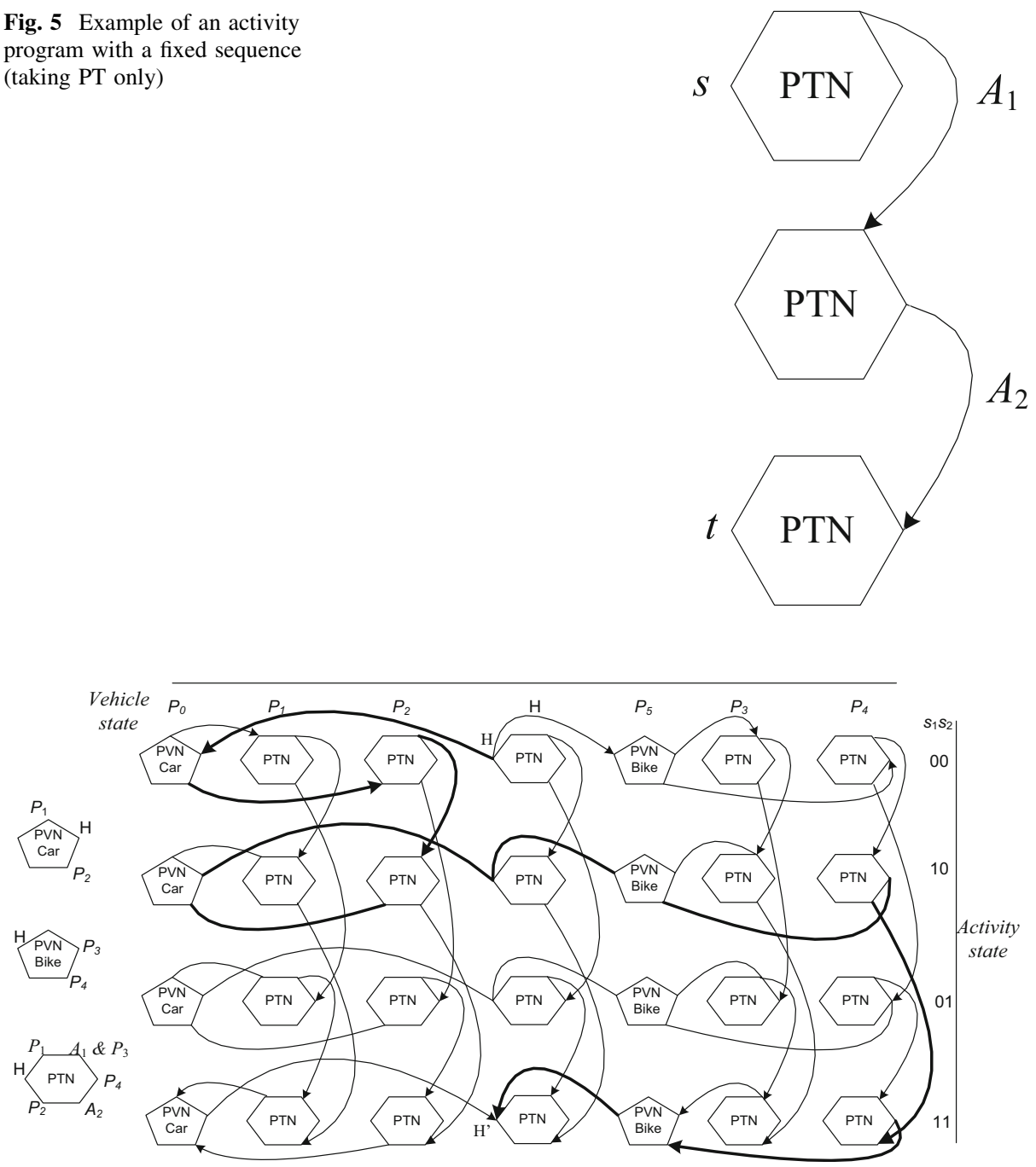

Fig. 6 Multi-state supernetwork representation. The path denoted by the bold links shows that the individual leaves home by car to conduct the fixed activity at $A_{1}$ with parking at $P_{2}$, then returns home and switches to bike to conduct the flexible activity at $A_{2}$ with parking at $P_{4}$, and finally returns home

$A_{2}$. Figure 6 displays the multi-state supernetwork representation of conducting an activity program with multiple activities with flexible sequence and multiple private vehicles. The activity program includes one fixed activity (at $A_{1}$ ) and one flexible activity (at $A_{2}$, assuming only one alternative location for the sake of simplicity), and two private vehicles (car and bike). $P_{1} \& P_{2}$ and $P_{3} \& P_{4}$ are parking locations for car and bike respectively. $P_{0}$ and $P_{5}$ denote that the car and bike respectively are in use (not parked). $s_{1} s_{2}$ represents the activity states for $A_{1} \& A_{2}$. Let $\mathrm{H}$ and $\mathrm{H}^{\prime}$ denote home at the start and end of the activity states respectively. It can be proven that any path from $\mathrm{H}$ to $\mathrm{H}^{\prime}$ denotes a possible full daily travel option or activity-travel pattern. Undirected links are bi-directed. (see Liao et al. 2010, 2011, 2013 for a detailed description.) 
Similarly, travel robustness can be evaluated by Eqs. 2-5 and 7-11. Those identified travel options that may be utilized to fulfill the activity program must satisfy the spacetime constraints (Chen and Kwan 2012; Liao et al. 2013). In Eq. 11, $\beta_{B}$ then becomes the maximum effort in terms of travel time, cost or GTC acceptable to conduct the activity programs.

\section{Illustration: a case study}

\section{Setup}

It is beyond the aim of this section to illustrate the full methodology and link it to the conceptualization as presented in "The proposed family of measures", "Operationalization of the measures" sections, nor to draw policy-relevant conclusions for the case study area. The aim is simply to illustrate that a real world implementation of the methodology is possible. Based on Eqs. 2-5 and 7-11, we calculate the number of travel options and the robustness indicators in a multi-modal transport context between the centers of ten cities in the Hague-Rotterdam-Dordrecht corridor (The Netherlands). Thus, we operationalize the accessibility measure for travel robustness in the diagram of Fig. 3, in which travel options involving car, PT and multi-modal are evaluated and generated simultaneously. For reasons of data availability, we only use travel times as calculated by our model, ignoring ranges due to variability over a day or week, and weather, etc. The outcomes could be of relevance for, for example, companies that are specialized in inner city deliveries, or policy-makers who aim to explore policies to increase the travel robustness of inner city areas. Based on the results companies specialized in inner city deliveries could, for example, include more additional time in their scheduling of deliveries for unexpected travel time delays: the fewer alternatives, the more additional travel time could be considered. Policy makers could use our results by putting more emphasis on developing parallel routes in case of relatively vulnerable OD pairs.

Figure 7 shows the cities and the road network of the study area. Consider three levels of roads, namely $<$ highway, regional, local $>$, with average speeds of $<90,50,30 \mathrm{~km} /$ $\mathrm{h}>$ by car respectively. 42 black dots denote the train stations in the corridor. The PT timetable is provided by a travel information company-OV9292 (www.ov9292.nl) in the year of 2011. Car drivers are allowed to park the cars at one of the train stations (also known as park and ride facilities) and continue the trips by PT. Travel time is taken as the main measure for travel options and $\beta_{B}$ is set as $60 \mathrm{~min}$, which is considered the maximum acceptable travel time in the corridor. We set the maximum travel time as twice the shortest travel time, and the overlapping ratio threshold as 0.2 . Assume that it takes $8 \mathrm{~min}$ to access and egress PT.

\section{Results: OD pairs}

Figure 8 illustrates the two travel options satisfying the two upper bounds by car only. From The Hague to Delft (Fig. 8a), the path P1 is the route with the shortest travel time, which is $14.8 \mathrm{~min}$, while the path $\mathrm{P} 2$ takes $19.3 \mathrm{~min}$. The overlapping ratio is 0 , which makes the total robustness indicator 1.50 according to Eq. 11. Note that a person travelling from the Hague to Delft could switch from route P1-P2 or vice versa about half way, but the two additional paths are not included because these violate the overlapping ratio 


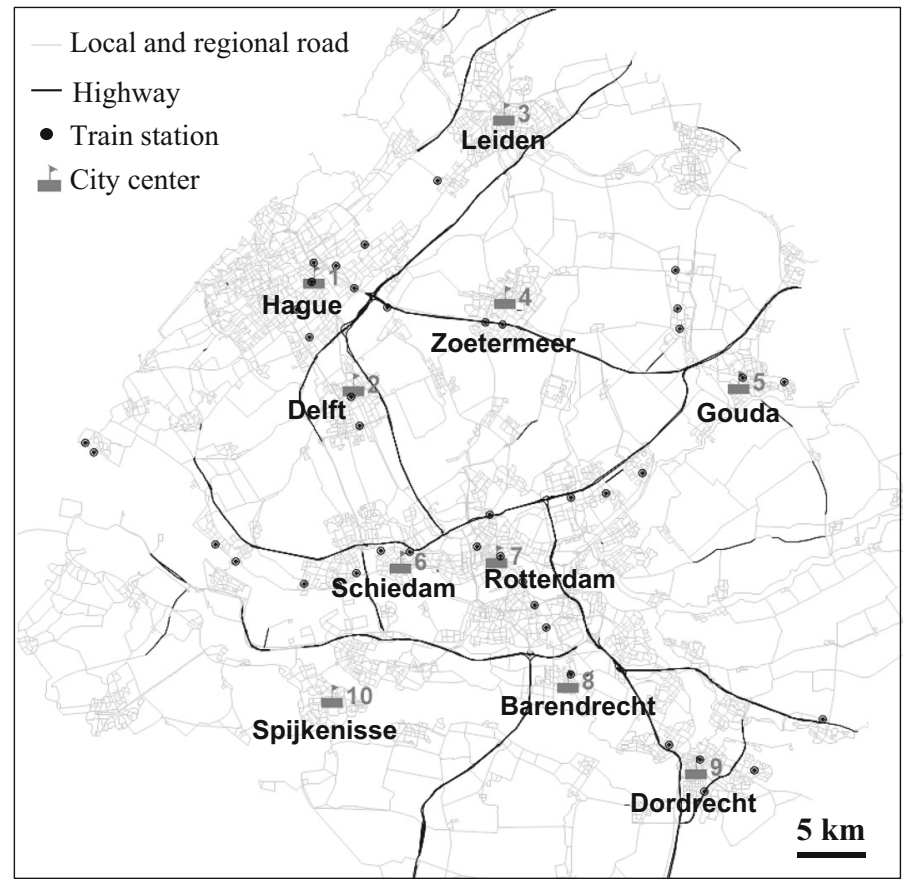

Fig. 7 The study area

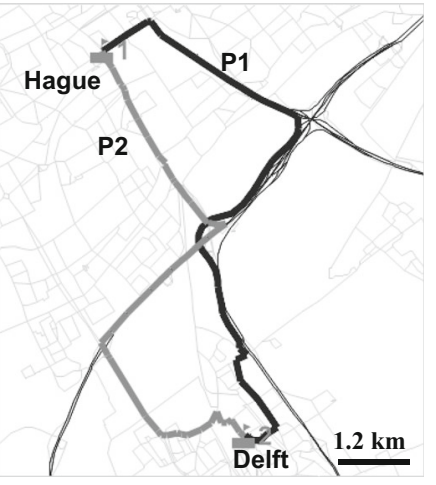

(a)

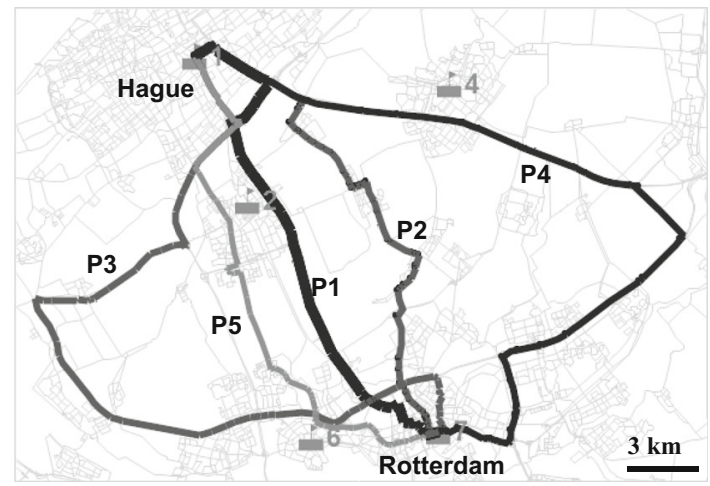

(b)

Fig. 8 Travel options by car only. a Hague to Delft, b Hague to Rotterdam

threshold of 0.2 (see above). Likewise, Fig. $8 \mathrm{~b}$ illustrates the five travel options from Hague to Rotterdam by car only. The path P1 is the route with the shortest travel time, which is $25.6 \mathrm{~min}$, while the longest travel time is $47.3 \mathrm{~min}$ denoted by the path P5. The maximum overlapping ratio between any two paths is 0.174 . The robustness indicator between the two cities is 2.34 .

Figure 9 shows the travel options by further considering PT and multi-modal trips, i.e., car plus PT. From The Hague to Delft (Fig. 9a), an intercity train connection and a 


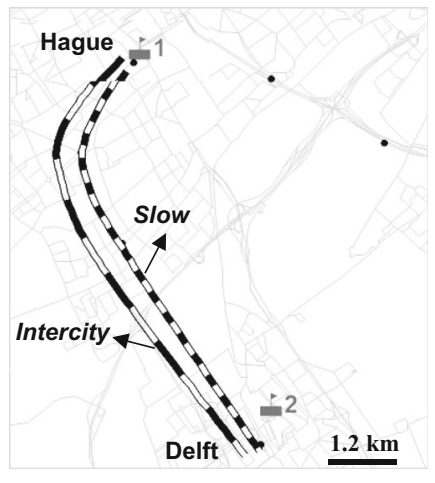

(a)

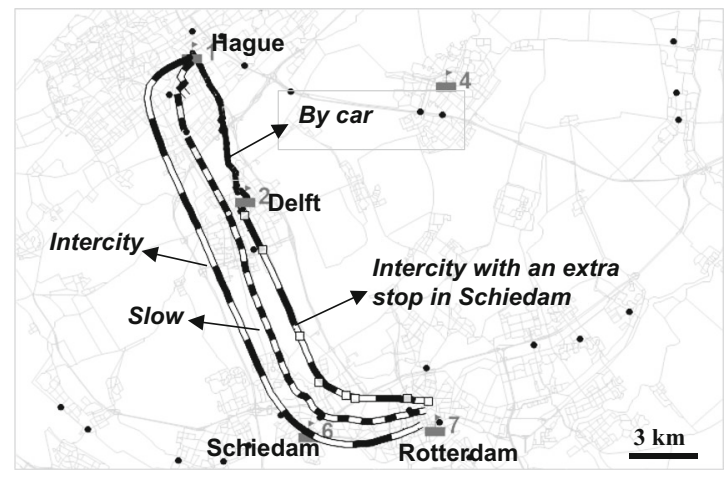

(b)

Fig. 9 Travel options by PT and multi-modal. a The Hague to Delft, b The Hague to Rotterdam

relatively slow one with intermediate stops are further added up to the set of travel options. It is assumed that slow train disruptions do not affect intercity trains, and vice versa, although in practice they may partly use the same tracks. Constrained by the two upper bounds, there are no valid multi-modal travel options due to the short distance between the two cities and the relatively long access/egress time. Nevertheless, for trips between The Hague and Rotterdam, multi-modal travel creates an option (Fig. 9b). To avoid overlap, the multi-modal trip utilizes a sub-path by car slower than P1 and P2 in Fig. 8a from The Hague to Delft and thereby switches to an intercity train connection with an extra stop in Schiedam, in total taking $50.3 \mathrm{~min}$. Taken together, there are 4 and 8 travel options from The Hague to Delft and Rotterdam with robustness indicator of 3.05 and 4.11 respectively.

\section{Results for the study area}

With the same setup of "Setup" section, Table 1 shows the values of the travel robustness indicator (Eq. 11) and the number of travel options between the cities. The higher the score, the more options travelers have available. As shown, the robustness indicators and the number of travel options differ significantly between OD pairs. The robustness indicator ranges from 1.16 (i.e., from Leiden to Spijkenisse) to 4.76 (i.e., from The Hague to Schiedam), while the number of travel options ranges from 2 to 9 . Due to the travel time/ distance difference, some OD pairs have different robustness indicators in spite of the same number of travel options.

It should also be noted that multi-modal trips may create extra travel options for an OD pair, but maybe not or less so for the opposite direction, since the PT services and the locations for change of modes (e.g., train stations) may not be evenly distributed. In the study area, this does not significantly apply. And if a person travels to a public transport node (e.g. a station) by car or bicycle, that person must return to the same node and travel back using the car or bicycle again. Since we calculated the results only for one direction (and not the return trip) this is not relevant for our example. Note that this is not an issue for evaluating the travel robustness of conducting an activity program (Sect. 3.3 and Fig. 6) because multi-modal trips are chained as a part of a full activity-travel pattern (path) and evaluated against other patterns. 


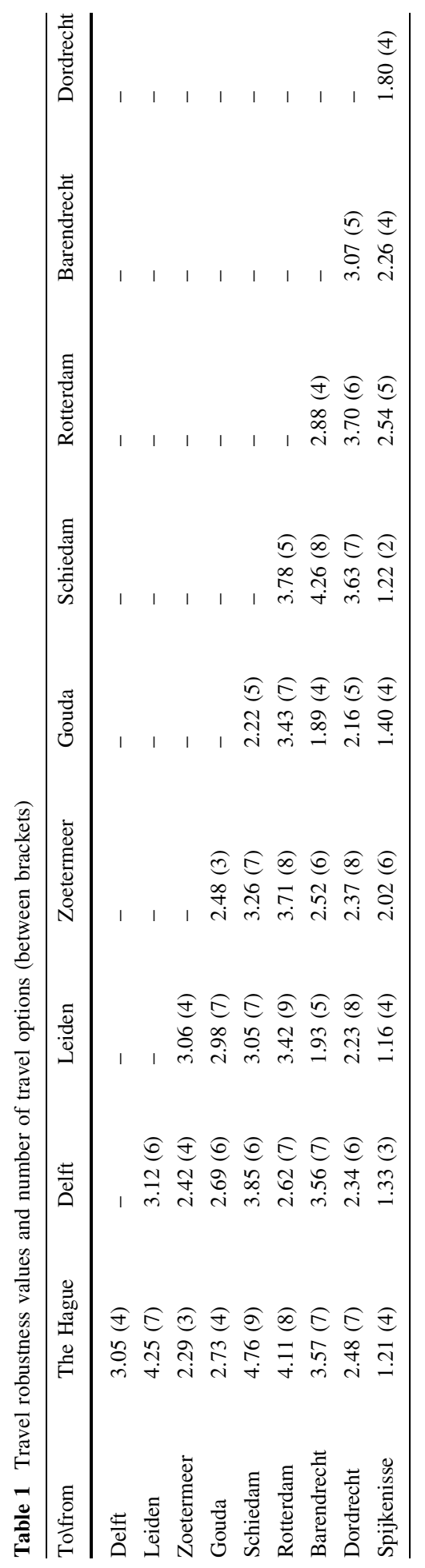




\section{Sensitivity analysis}

Sensitivity analyses are conducted on the two parameters concerning the two upper bounds by examining the travel options from The Hague to Rotterdam.

First, the travel time upper bound is kept the same, i.e., two times the shortest travel time, whereas the overlapping ratio varies from 0.1 to 0.5 with a step size of 0.1 . Figure 10 shows the results that, as expected, the values of robustness indicator increase if more overlap is allowed. The robustness indicator increases more slowly than the number of travel options following Eq. 11 because the added value of an increase in the number of travel options is probably subject to the law of diminishing returns. This is because the chance of disruptions in multiple routes simultaneously is relatively small. Likewise, the chance of benefiting from a large number of options is limited. We expect the indicator for robustness is probably more relevant for decision-making than the number of travel options. In the second sensitivity analysis, we keep the overlapping upper bound the same, i.e., 0.2, whereas, travel time upper bound changes from 1.2 to 2.8 with 0.4 as the step size. Figure 11 shows that the choice for the ratio up to 2.4 has a large impact on the robustness indicator and the number of travel options, but higher ratios have little further improvement.

Our sensitivity analyses show the importance of carefully setting the threshold values for both parameters. However, it is beyond the aim of this paper to discuss which values are the most appropriate.
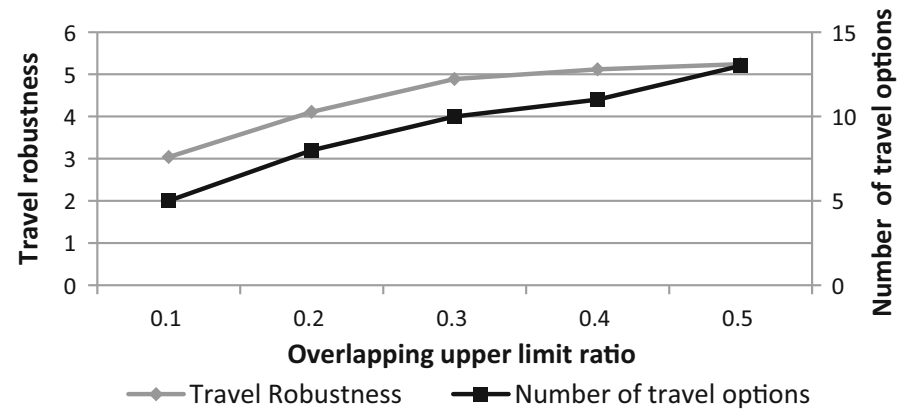

Fig. 10 Impact of overlapping ratio on robustness and the number of travel options

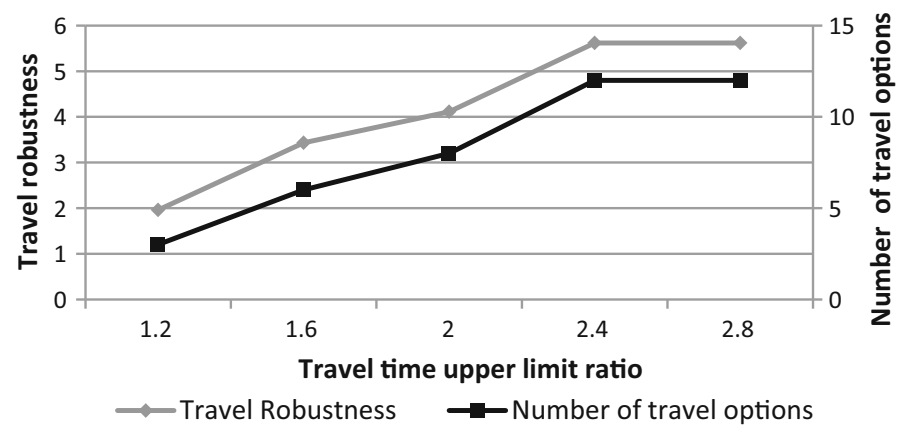

Fig. 11 Impact of maximum travel time on robustness and the number of travel options 


\section{Conclusions and future work}

This paper represents a general attempt to put a new family of accessibility measures for travel robustness on the research agenda. The measures need to be developed further, and applied in real world cases. In this section, we therefore not only present the main conclusions, but also discuss avenues for further development, valuation of outcomes, and policy relevant applications.

We argue that the robustness of the transport system, conceptualized via the number of travel options between origins and destinations, can be seen as an accessibility measure. As discussed in the introduction, infrastructure-based measures are often presented as accessibility measures, even though they only include the resistance component but not the options to reach destinations. Following this line of literature, our measures can be labeled as accessibility measures as well. A higher score on our measures contribute to more options for activity participation or reaching destinations. We have developed a methodology to operationalize the measures expressing options in terms of network representations for multi-modal travel and activity participation. There are several choices that need to be made to operationalize the measures in specific indicators. Choices relate to the overlap between candidate options, mode(s) to be included, origins and destinations to be included and mathematical expressions. Our methodology makes use of concepts from time geography and path choice modeling, but to the best of our knowledge we are the first to introduce these as accessibility measures of the robustness of the transport system. Such a family of accessibility measures can be very relevant for policy making, including comparisons of geographical areas, for the robustness of the transport system of an area over time and the impact of policy measures on robustness.

Nevertheless, several issues are still worth consideration in future research.

\section{Options to extend our conceptualization}

In line with the discussion in "The proposed family of measures" section, first of all, more comprehensive methods to correct for overlap in travel options need to be developed. Secondly, options, including levels of flexibility with respect to location choices, time constraints of preferences, and activity orders, need to be developed. Thirdly, we almost completely ignored the links between ICT and accessibility, but these will very likely become increasingly important. For example, e-shopping may increasingly become a substitute for visiting shops (Farag et al. 2007). Fourthly, it makes sense to not simply count the number of opportunities, but to weight them according to how attractive they are. For example, a small supermarket with relatively high prices is not a complete substitute for a large supermarket with lower prices. Fifthly, our methodology does not cover all the concepts for GTC that are relevant and presented in the introduction. This paper includes the availability of multiple paths, but not the variability and predictability of travel time or GTC of given paths. Sixthly, we propose one mathematical specification of the measure for robustness with an exponential form. However, other specifications are also possible. We recommend empirical research find out which specification best matches the preferences of travelers.

\section{Valuation of robustness}

The traditional way of valuing the benefits of new infrastructure in a Cost-Benefit Analysis (CBA) framework is to value travel time gains for existing travelers, and to value induced 
travel via the rule of half. This method is not suitable for the evaluation of having multiple options available because the benefits are not translated only into travel times. The increasingly more popular logsum method is not perfectly suitable because it does not explicitly include the value of having more options for the travelers. There are a few studies on the valuation of variability, delays, etc. that can be of use. The main benefits of increasing travel options are that travel times will become more reliable. Therefore, conventional methods to value variability can be useful if the link between increasing travel options and reducing variability can be established. As long as the evaluations include many origins and destinations, rules of thumb can be applied for this link as long as these rules more or less reflect the average valuations. We give a brief overview of the literature showing options to value travel time variability. The valuation of the variability of travel times are based on the distributions of travel times (e.g. Lam and Small 2001), but a problem is that transferability is limited because the distributions are context dependent. Sikka and Hanley (2013) used the frequency of delay days to measure traveler's sensitivities to travel time reliability. An advantage is that this method is relatively simple. They found that travelers are both averse to the amount of unexpected delay as well as to the frequency of days with unexpected delays. Tseng et al. (2012) developed a method to value anticipating behavior for train delays. To summarize, some options exist to value the impact of travel options via the impact on travel time variability. Nevertheless, more research is needed to understand how much and for what reasons travelers value having multiple travel options.

\section{Interpretation of results: the importance of components of robustness}

We realize that a high score can result from multiple travel routes with little overlap to a narrow set of destinations, or from a limited set of travel routes to a wider range of destinations. The latter is arguably of more importance to individuals than the former. Consequently, we think that future work could focus on disentangling components of robustness and linking them to options to reach destinations or to participate in activities.

\section{Policy relevant applications}

In placing our proposal for a new family of accessibility measures in the policy context, policy-makers should be interested in the value travelers attribute to having multiple travel options available. This is because it is usual to apply a broad welfare approach-this is very common that many countries evaluate transport policy options, at least options for new infrastructure, using a CBA framework (Hayashi and Morisugi 2000), and in such a framework a broad welfare perspective is taken. If the accessibility effects are included in ex-ante or ex-post policy evaluations, an important issue is how to communicate the outcomes of accessibility measures. Above we argued that a trade-off exists between ease of communication and the behavioral realism of measures. More research is needed to find out how best to select the measures and communicate the results to policy-makers.

Open Access This article is distributed under the terms of the Creative Commons Attribution 4.0 International License (http://creativecommons.org/licenses/by/4.0/), which permits unrestricted use, distribution, and reproduction in any medium, provided you give appropriate credit to the original author(s) and the source, provide a link to the Creative Commons license, and indicate if changes were made. 


\section{References}

Arentze, T.A., Timmermans, H.J.P.: A multi-state supernetwork approach to modelling multi-activity multimodal trip chains. Int. J. Geogr. Inf. Sci. 18, 631-651 (2004)

Bates, J., Polak, J., Jones, P., Cook, A.: The valuation of reliability for personal travel. Transp. Res. Part E 37(2-3), 191-229 (2001)

Bekhor, S., Ben-Akiva, M.E., Ramming, S.: Evaluation of choice set generation algorithms for route choice models. Ann. Oper. Res. 144(1), 235-247 (2006)

Bertolini, L., Curtis, C., Renne, J.: Station area projects in Europe and beyond: towards transit oriented development? Built Environ. 38(1), 31-50 (2012)

Börjesson, M., Eliasson, J.: On the use of “Average Delay" as a measure of train reliability. Transp. Res. Part A 45(3), 171-184 (2011)

Bovy, P.H.L., Fiorenzo-Catalano, S.: Stochastic route choice set generation: behavioral and probabilistic foundations. Transportmetrica 3(3), 173-189 (2007)

Carlier, K., Fiorenzo-Catalano, S., Lindvel, C., Bovy, P.: A supernetwork approach towards multimodal travel modelling. Proceedings of the 82 nd annual meeting of the transportation research board, Washington, D.C (2003)

Cats, O., Jenelius, E.: Planning for the unexpected: the value of reserve capacity for public transport network robustness. Transp. Res. Part A 81, 47-61 (2015)

Caschili, S., Medda, F.R., Reggiani, A.: Guest editorial: resilience of networks. Transp. Res. Part A. 81, 1 (2015). doi:10.1016/j.tra.2015.07.010

Chen, A., Yang, H., Tang, W.H.: Capacity reliability of a road network: an assessment methodology and numerical results. Transp. Res. Part B 36(3), 225-252 (2002)

Chen, X., Kwan, M.-P.: Choice set formation with multiple flexible activities under space-time constraints. Int. J. Geogr. Inf. Sci. 26(5), 941-961 (2012)

Chorus, C., de Jong, G.C.: Modeling experienced accessibility for utility-maximizers and regret-minimizers. J. Transp. Geogr. 19(6), 1155-1162 (2011)

De Jong, G., Daly, A., Pieters, M., van der Hoorn, T.: The logsum as an evaluation measure: review of the literature and new results. Transp. Res. Part A. 41(9), 874-889 (2007)

D'Lima, M., Medda, F.: A new measure of resilience: an application to the London underground. Transp. Res. Part A. 81, 35-46 (2015)

Farag, S., Schwanen, T., Dijst, M., Faber, J.: Shopping online and/or in-store? A structural equation model of the relationships between e-shopping and in-store shopping'. Transp. Res. A. 41(2), 125-141 (2007)

Geurs, K., Haaijer, R., Van Wee, B.: Option value of public transport: methodology for measurement and case study for regional rail links in The Netherlands. Transp. Rev. 26(5), 613-643 (2006)

Geurs, K.T., van Wee, B.: Accessibility evaluation of land-use and transport strategies: review and research directions. J. Transp. Geogr. 12(2), 127-140 (2004)

Guihaire, V., Hao, J.-K.: Transit network design and scheduling: a global review. Transp. Res. Part A. 42(10), 1251-1273 (2008)

Guo, Z., Wilson, N.H.M.: Assessing the cost of transfer inconvenience in public transport systems: a case study of the london underground. Transp. Res. Part A. 45(2), 91-104 (2011)

Hayashi, Y., Morisugi, H.: International comparison of background concept and methodology of transportation project appraisal. Transp. Policy. 7(1), 73-88 (2000)

Janic, M.: Reprint of "Modelling the resilience, friability and costs of an air transport network affected by a large-scale disruptive event”. Transp. Res. Part A. 81, 77-92 (2015)

Koopmans, C., Groot, W., Warffemius, P., Annema, J.A., Hoogendoorn-Lanser, S.: Measuring generalised transport costs as an indicator of accessibility changes over time. Transp. Policy. 29, 154-159 (2013)

Laird, J., Geurs, K., Nash, C.: Option and non-use values and rail project appraisal. Transp. Policy. 16(4), 173-182 (2009)

Lam, T.C., Small, K.A.: The value of time and reliability: measurement from a value pricing experiment. Transp. Res. Part E. 37(2-3), 231-251 (2001)

Levine, J.: Zoned Out: Regulation, Markets, and Choices in Transportation and Metropolitan Land Use. RFF Press, Washington DC (2005)

Liao, F., Arentze, T.A., Timmermans, H.J.P.: Supernetwork approach for multi-modal and multi-activity travel planning. Transp. Res. Rec. 2175, 38-46 (2010)

Liao, F., Arentze, T.A., Timmermans, H.J.P.: Constructing personalized transportation network in multistate supernetworks: a heuristic approach. Int. J. Geogr. Inform. Sci. 25(11), 1885-1903 (2011)

Liao, F., Arentze, T.A., Timmermans, H.J.P.: Incorporating space-time constraints and activity-travel time profiles in a multi-state supernetwork approach to individual activity-travel scheduling. Transp. Res. Part B. 55, 41-58 (2013) 
Liao, F., Arentze, T.A., Molin, E.J.E., Bothe, W., Timmermans, H.J.P.: Effects of integrated land-use transport scenarios on travel patterns: a multi-state supernetwork application. Transportation (2016). doi:10.1007/s11116-015-9616-Z

Lyons, G., Urry, J.: Travel time use in the information age. Transp. Res. Part A. 39(2-3), 257-276 (2005)

Mazloumi, E., Currie, G., Rose, J.: Using GPS data to gain insight into public transport travel time variability. J. Transp. Eng. 136(7), 623-631 (2010)

Ministerie van Infrastructuur en Milieu: Structuurvisie Infrastructuur en Ruimte. Ministerie van Infrastructuur en Milieu, The Hague (2012)

Mokhtarian, P.L.: Telecommunications and travel: the case for complementarity. J. Ind. Ecol. 6(2), 43-57 (2002)

Neutens, T., Witlox, F., Van De Weghe, N., De Maeyer, P.H.: Space-time opportunities for multiple agents: a constraint-based approach. Int. J. Geogr. Inf. Sci. 21(10), 1061-1076 (2007)

Pimm, S.L.: The Balance of Nature. University of Chicago Press, Chicago (1991)

Prato, C.G.: Route choice modeling: past, present and future research directions. J. Choice Model. 2(1), 65-100 (2009)

Pyrga, E., Schulz, F., Wagner, D., Zaroliagis, C.: Efficient models for timetable information in public transportation systems. ACM. J. Exp. Algorithm. 12, 1-39 (2008)

Reggiani, A., Nijkamp, P., Lanzi, D.: Transport resilience and vulnerability: the role of connectivity. Transp. Res. Part A. 81, 4-15 (2015)

Rietveld, P., Bruinsma, F.R., Van Vuuren, D.J.: Coping with unreliability in public transport chains: a case study for Netherlands. Transp. Res. Part A. 35(6), 539-559 (2001)

Sikka, N., Hanley, P.: What do commuters think travel time reliability is worth? Calculating economic value of reducing the frequency and extent of unexpected delays. Transportation 40(5), 903-919 (2013)

Tseng, Y.-Y., Rietveld, P., Verhoef, E.T.: Unreliable trains and induced rescheduling: implications for costbenefit analysis. Transportation 39(2), 387-407 (2012)

Van Wee, B., Bohte, W., Molin, E., Arentze, T., Liao, F.: Policies for synchronization in the transport-landuse system. Transp. Policy. 31(1), 1-9 (2014)

Van Wee, B., Geurs, K., Chorus, C.: Information, communication, travel behavior and accessibility. J. Transp. Land. Use. 6(3), 1-16 (2013)

Yen, J.Y.: Finding the K shortest loopless paths in a network. Manage. Sci. 17, 712-716 (1971)

Feixiong Liao is currently an assistant professor at the Urban Planning Group, Eindhoven University of Technology, the Netherlands. His research interests include activity-based modelling, accessibiity analysis, discrete choice modelling, and supernetwork modelling for applications in transportation research and urban planning.

Bert van Wee is professor of transport policy at Delft University of Technology, the Netherlands, and scientific director of TRAIL research school, the Netherlands. 